\title{
The Time Delay in the Gravitational Lens PKS 1830-211
}

\author{
J.E.J. Lovell ${ }^{1,2}$, D.L. Jauncey ${ }^{3}$, J.E. Reynolds ${ }^{3}$, M.H. Wieringa ${ }^{3}$, E.A. King, ${ }^{3,4}$, A.K. Tzioumis ${ }^{3}$, \\ P.M. McCulloch ${ }^{1}$, P.G. Edwards ${ }^{5}$
}

\begin{abstract}
We have measured a time delay of $26_{-5}^{+4}$ days and a magnification ratio of $1.52 \pm 0.05$ in the strong radio gravitational lens PKS 1830-211. The observations were made over the 18 month period from 1997 January to 1998 July with the Australia Telescope Compact Array at $8.6 \mathrm{GHz}$, and have shown that the source started a large flux density outburst around 1997 June.
\end{abstract}

Subject headings: galaxies: individual (PKS 1830-211) — Gravitational lensing

\footnotetext{
${ }^{1}$ School of Mathematics and Physics, University of Tasmania, Hobart, Tasmania 7001, Australia

${ }^{2}$ Now at Institute of Space and Astronautical Science, Sagamihara, Kanagawa 229-8510, Japan

${ }^{3}$ Australia Telescope National Facility, CSIRO, Epping, New South Wales 2121, Australia

${ }^{4}$ Present address CSIRO Earth Observation Centre, Canberra, ACT, 2601, Australia

${ }^{5}$ Institute of Space and Astronautical Science, Sagamihara, Kanagawa 229-8510, Japan
} 


\section{Introduction}

A precise measurement of the time delay between the images in a gravitational lens is essential if it is to fulfill its potential as a tool for estimating the Hubble constant. The flat-spectrum radio source PKS 1830-211 (Rao \& Subrahmanyan 1988) was found to be an Einstein ring/gravitational lens, comprised of two compact, flat-spectrum components located on opposite sides of a 1 arcsec diameter ring (Jauncey et al. 1991). It is by far the strongest radio lens yet found, being $\sim 10 \mathrm{Jy}$ at $4.8 \mathrm{GHz}$. Observations of molecular absorption at centimeter and millimeter wavelengths have revealed two intervening galaxies at $z=0.19$ (Lovell et al. 1996a) and $z=0.89$ (Wiklind \& Combes 1996). VLBI observations of the two compact lensed components have revealed striking structural differences on milliarcsecond scales (Garrett et al. 1996) suggesting that both galaxies may be involved in the lensing (Lovell et al. 1996a). PKS 1830-211 varies dramatically at radio wavelengths (Lovell et al. 1996b) and VLBI observations show that the variations are confined to the two compact components (King 1994), making it an excellent candidate for relative time delay measurements.

This paper describes our measurements with the $6 \times 22 \mathrm{~m}$ Australia Telescope Compact Array (ATCA) to determine the lensing time delay in PKS 1830-211 through a correlation of the flux density light curves of its compact components. Section 2 describes the development of our observing strategy and data reduction, section 3 describes the analysis of our data to obtain a time delay and magnification ratio. In section 4 we describe our error analysis and in section 5 we discuss the implications of our measurements on modeling the PKS 1830-211 lensing system.

\section{Observations and Data Reduction}

The presence of a single peak in the $8.4 \mathrm{GHz}$ total flux density light curve in early 1992 (Lovell et al. 1996b) indicates that the lensing time delay is less than a few months (a time delay that was much longer would result in a broader or double-peaked light curve). Consequently, in order to sufficiently time resolve these variations in the compact components it was necessary to measure the flux densities of the two components separately on timescales shorter than both the total flux density variations and the lensing time delay, previously estimated to be $44 \pm 9$ days (van Ommen et al. 1995). We commenced our ATCA monitoring observations in 1995 August, making observations at approximately monthly intervals. The two compact and variable components can be measured separately, as explained below, on the $6 \mathrm{~km}$ East-West array of the ATCA at 8.6 GHz if observations are restricted to between 15 and 19 hours LST when the ATCA provides sub-arcsecond spatial resolution along the position angle of the compact components. The strong, compact, flat-spectrum source PKS 1921-293 was observed in each session to provide the relative gain calibration of the individual antennas, while overall flux density calibration was determined through observations of the ATCA primary flux density calibrator PKS 1934-638. The total flux density variations of PKS 1830-211 will be presented in a future paper (Lovell et al. 1998, in 
preparation). During the period 1996 July 26 through 1996 August 8, a more closely spaced series of observations was undertaken in order to gain an understanding of the shorter-term variability. Analysis of this 1996 July-August data showed more rapid variability than previously thought, so from 1997 January, one observation every three to six days was made to better sample the light curves. In this paper we restrict our analysis to the data from 1996 July onwards, although the data from the period 1996 December 20 through 1997 January 10 were also excluded as the source was too close to the sun.

As the two compact components of PKS 1830-211 are not fully resolved from the ring at the $\sim 0.9$ arcsecond resolution of the ATCA at $8.6 \mathrm{GHz}$, we have adopted a model fitting approach to determine the flux densities of the individual components. We chose a simple model consisting of two circular Gaussian components, each of which also contains a contribution from the non-varying ring. The component separation was set initially to 0.95 arcseconds at a position angle of 46 degrees, as determined from VLBI observations (King 1994). These values were allowed to change slightly during model fitting, but were found to deviate by no more than 3 degrees in position angle and 12 mas in separation. All data were edited and calibrated using AIPS before being exported to Difmap (Shepherd 1997) for model fitting.

To verify that this model successfully describes the source, we analyzed an edited section from a full ATCA synthesis observation taken on 1994 December 19 when PKS 1830-211 had a total flux density of 7.2 Jy. We found that the individual fitted Gaussian component flux densities differed by no more than $70 \mathrm{mJy}$ from those measured from the full synthesis data. As a further check, simulated ATCA data sets were generated and analyzed using the above procedures. For all of these the modeled component flux density changes were accurate to within 1\%, and the total flux density in the model accounted for $99.8 \%$ of the total simulated flux density. This clearly demonstrates the robustness and reliability of our model fitting procedures in determining small changes in each of the compact components of PKS 1830-211.

Component flux density errors were estimated at each epoch using the method described by Tzioumis et al. (1989) and were found to be typically $50 \mathrm{mJy}$. The NE and SW component flux density light curves obtained from the fitted 8.6 GHz ATCA data since 1996 January are shown in Figure 1(a). We have detected clear changes in the flux densities of the two components, with a roughly linear increase seen to begin sometime between MJD 50530 and MJD 50670. There is a clear difference in gradient between the two light curves, which is a result of different image magnifications. The figure also shows some significant differences in the light curves, the most prominent being a distinct 'bump' of 400 mJy in the NE component light curve near MJD 50700 that is seen to occur significantly later in the SW light curve, indicating a time delay of 20 to 30 days. In the following section, we discuss a detailed analysis of the data to obtain a quantitative estimate of the time delay and magnification ratio and their uncertainties. 


\section{Analysis}

To estimate the time delay it is important to consider how the variability of the lensed source is detected by the observer. In the following analysis, the subscripts 1 and 2 refer to the NE and SW components of PKS 1830-211 respectively. We describe the time variation of the NE and SW compact components of the lensed image as $S_{1}(t)$ and $S_{2}(t)$ respectively. The SW component light curve is expected to be identical to the NE light curve but magnified by a different amount and shifted in time, thus:

$$
S_{2}(t)=\frac{1}{\mu} S_{1}(t+\Delta \tau)
$$

where $\Delta \tau$ is the time delay and $\mu$ is the relative magnification ratio. Also lensed is an extended

component that forms the Einstein ring which contributes a constant flux density $S_{\text {ring }}$. The total observed flux density can therefore be described as

$$
S_{m}(t)=S_{1}(t)+S_{2}(t)+S_{\text {ring }}
$$

The Einstein ring contributes a large proportion, up to $50 \%$ of the total flux density at $\mathrm{cm}$ wavelengths (Jauncey et al. 1991). Therefore the effect of the ring flux density must be considered before attempting to determine a lensing time delay. In our ATCA observations, neither $S_{1}(t)$ nor $S_{2}(t)$ are measured directly, instead the measured quantities are

$$
S_{m 1}(t)=S_{1}(t)+S_{c 1}
$$

and

$$
S_{m 2}(t)=S_{2}(t)+S_{c 2}=\frac{1}{\mu} S_{1}(t+\Delta \tau)+S_{c 2}
$$

where the quantities $S_{c 1,2}$ are constant but unknown and $S_{\text {ring }}=S_{c 1}+S_{c 2}$. It is unlikely that $S_{c 1}$ and $S_{c 2}$ are equal. Therefore it is also necessary to solve for $d$, the difference between $S_{c 1}$ and $S_{c 2}$. The two light curves may now be compared using trial values of $\mu, \Delta \tau$ and $d$ so that a solution for the relative magnification ratio and the time delay can be obtained.

\subsection{Dispersion Analysis}

A correlation of the two light curves was undertaken using the dispersion analysis method introduced by Pelt et al. $(1994,1996)$ to analyze the component light curves of the gravitational lens $0957+561$. The dispersion method has been shown to be successful for $0957+561$, which has a relatively large time delay of $417 \pm 3$ days (Kundić et al. 1997). We chose this method because

it avoids any interpolation between data points. Methods that are based on interpolation can lead to erroneous results as they put equal weight on assumed and measured data.

Following Pelt et al., for every test value of $\mu, \Delta \tau$ and $d$, two light-curve datasets $a_{i}$ and $b_{i}(i=1, \ldots, N$ where $N$ is the number of observations $)$ are obtained. Together they form a 
combined light-curve where $a_{i}$ contains the NE variable component data and $b_{i}$ contains the SW variable component data modified in flux density and time by the test values for $\mu, \Delta \tau$ and $d$. The dispersion, $D^{2}$, of this combined light curve is calculated from the weighted sum of squared differences between nearby $a_{i}, b_{i}$ pairs over the entire curve:

$$
D^{2}(\Delta \tau, \mu)=\frac{\Sigma_{i j} W_{i j} V_{i j}^{\prime}\left(a_{i}-b_{j}\right)^{2}}{2 \Sigma_{i j} W_{i j} V_{i j}^{\prime}}
$$

where

$$
V_{i j}^{\prime}= \begin{cases}1 & \text { if }\left|t_{i}-t_{j}\right| \leq \delta, \\ \left(1+\left(\frac{\delta-\left|t_{i}-t_{j}\right|}{\delta / 2}\right)^{2}\right)^{-1} & \text { if }\left|t_{i}-t_{j}\right|>\delta .\end{cases}
$$

and $W_{i}=1 / e_{i}^{2}$ where $e_{i}$ is the standard error in data point $i$. The weighting factor $V_{i j}^{\prime}$ ensures that data pairs are only given significant weighting if they are less than $\sim \delta$ days apart and is modified from the $V_{i j}$ of Pelt et al. to avoid strong weighting on data points with near-zero separation. These points add a bias towards a zero delay for relatively short time delays, as we expect to find in PKS 1830-211. The values of $\Delta \tau, \mu$ and $d$ for which $D^{2}$ is a minimum are thus the best estimate of the time delay, magnification ratio and constant flux density offset difference.

Before a correlation of the light curves can be made, an estimate of $\delta$, effectively the longest timescale over which the source is believed not to show significant variability, must be determined. An inspection of the light curves in Figure 1, shows significant changes in flux density on timescales of the order of 10 days. We note that $V^{\prime}$ drops quite slowly, reaching 0.5 at a point separation of $1.5 \delta$. Therefore, we chose a value of $\delta=5$, slightly less than the estimated timescale, so that points with separations greater than $\sim 8$ days did not unduly influence the solution.

A single, unambiguous solution was found for $\delta=5$ days at $\Delta \tau=-23$ days (i.e. $\mathrm{NE}$ component leading), $\mu=1.52$ and $d=-0.62 \mathrm{Jy}$. We also investigated how the solution behaved for a range of $\delta$ and found that for $\delta<7$ days, $\Delta \tau$ stayed within \pm 4 days, $\mu$ stayed within \pm 0.02 and $d$ stayed within \pm 0.06 Jy of the $\delta=5$ days solution. For $\delta \geq 7$ days, up to $\delta=14$ days (the largest value we trialed), the solutions for $\Delta \tau$ became shorter, ranging from -12 to -17 days, but solutions for $\mu$ and $d$ changed little. A visual inspection of the combined light curves with these solutions applied clearly shows a poorer correlation than seen for $\delta<7$.

It is likely that this sudden change in the delay solution is due to an effective smoothing of the features that help register the light curves in delay. However, $\mu$ and $d$ are affected to a lesser degree as the overall smooth, rising light curves constrain these parameters well, and the short timescale changes have little influence. 


\section{Error Analysis}

We carried out Monte Carlo simulations to estimate the errors in our derivation of the time delay and magnification ratio. Five hundred light curve pairs were created with data points at the same epochs of the actual observations. The flux density of each point was calculated using a random number generator but constrained such that each simulated point was within the uncertainty of the original measurement. An additional constraint forced the sum of a pair of same-epoch measurements to be equal to the total measured flux density within the uncertainty of that observation.

Each of the 500 light curve pairs were then correlated to obtain estimates for $\Delta \tau, \mu$ and $d$ when $\delta=5$ days. We found that the correlated values of $\mu$ and $d$ were distributed in a Gaussian-like manner about the values obtained from our observations. We have used these distributions to estimate errors for $\mu$ and $d$ by measuring the width of the distributions at half their peak. We thus estimate that the magnification ratio, $\mu$, is $1.52 \pm 0.05$ and the flux density offset, $d$, is $-0.62 \pm 0.05 \mathrm{Jy}$. The distribution of $\Delta \tau$ from the simulations however is not distributed in a Gaussian-like fashion. Instead, a broad distribution of solutions between -12 and -30 days was obtained with peaks near $-15,-23$ and -28 days.

Eighteen years of monitoring the gravitational lens $0957+561$ have demonstrated that non-varying or smooth light curves contributes little information to a time delay solution; in fact such data can lead to ambiguous solutions. The detection of strong features in both light curves is required to obtain a reliable time delay measurement (Kundić et al. 1997). Bearing this in mind, we have also analyzed the subset of data surrounding the 'bump' seen near MJD 50700 where the most prominent and best sampled feature occurs (Figure 2(a)), specifically to avoid ambiguities in the time delay estimate.

We found a solution at $\Delta \tau=-26$ days, $\mu=1.68$ and $d=-1.17$ Jy for $\delta=5$ (Figure 2(b)). As expected, Monte Carlo simulations show the magnification ratio and flux density offset to be less well constrained here than with the full dataset. We have therefore adopted the values for $\mu$ and $d$ as determined from the full dataset as our best estimates. However, the simulations show the time delay for this subset to be well constrained without ambiguities with a Gaussian-like distribution of solutions around -26 days. By measuring the width of this distribution at half its peak, we have estimated an uncertainty and obtain a time delay of $-26_{-4}^{+5}$ days.

We are continuing our ATCA monitoring observations. When the current trend of increasing flux density at $8.6 \mathrm{GHz}$ ends, the change in gradient may provide even better constraints on the time delay. 


\section{Discussion}

Out ATCA monitoring observations have allowed us to measure the time delay and magnification ratio of the gravitational lens PKS 1830-211, providing new constraints for lensing models of this system.

Our time delay measurement is not in agreement with the $-44 \pm 9$ day measurement of van Ommen et al. (1995) from VLA monitoring observations at 8.6 and $15 \mathrm{GHz}$. We believe van Ommen et al. did not correctly account for the contribution of the Einstein ring flux density when calculating the magnification ratio. The difference between the total flux density of the source and the sum of the compact component flux densities fitted to the data, i.e. the contribution from the ring, should be constant. However in van Ommen et al.'s analysis the inferred Einstein ring contribution depends on the observing frequency and array configuration. We feel confident that our analysis of the ATCA data has correctly accounted for the constant Einstein ring flux density.

Millimeter observations, in early 1996, by Frye, Welch, \& Broadhurst (1997), which clearly separate the core components of the lens and show no extended jet structure on $\sim 0.5$ arcsec scales, provide an estimate for $\mu$ of $1.14 \pm 0.06$. Also, Wiklind \& Combes (1998) measured the magnification ratio in the millimeter band on three occasions over 11 months and found that it changed between 1.0 and 1.8 with an uncertainty in each case of 0.1 . We note that PKS 1830-211 has shown strong variability at millimeter wavelengths in the past (Tornikoski et al. 1996) and that Wiklind \& Combes observed a decrease in total flux density from 1.5 to 0.93 Jy over their observations. Such variability could influence the measurement of the magnification ratio at a single epoch. Also, the likelihood of sub-components in the compact structure at these wavelengths, similar to those seen at $43 \mathrm{GHz}$ by Garrett et al. (1998), should be investigated as this too has an impact on the magnification ratio. More detailed monitoring of the compact components at millimeter wavelengths can provide a test of our magnification ratio measurement if the time delay effect is accounted for.

It is not yet possible to obtain an estimate for $\mathrm{H}_{0}$ from the PKS 1830-211 lensing system as the redshift of the lensed AGN is not known, and the lensing galaxies are not well parameterized. However, if we assume that the intervening galaxy at $z=0.19$ has a negligible gravitational affect on the system, then we can apply our time delay measurement to the model of Nair et al. (1993)

for a lensing galaxy at $z=0.89$ to put constraints on the redshift of the background source, $z_{s}$. If $\mathrm{H}_{0}$ is assumed to be between 50 and $100 \mathrm{kms}^{-1} \mathrm{Mpc}^{-1}$ and $\mathrm{q}_{0}=1 / 2$, then a time delay of $-26_{-4}^{+5}$ days implies $z_{s}>1.4$, with a value of $z_{s} \approx 2$ for $\mathrm{H}_{0}=75 \mathrm{kms}^{-1} \mathrm{Mpc}^{-1}$.

The Australia Telescope is funded by the Commonwealth Government for operation as a national facility managed by CSIRO. J.E.J.L. acknowledges the receipt of a JSPS Fellowship. 


\section{REFERENCES}

Frye, B., Welch, W. J., \& Broadhurst, T. 1996, ApJ, 478, L25

Garrett, M., Nair, S., Porcas, R., \& Patnaik, A. 1996, in IAU 173: Astrophysical Applications of Gravitational Lensing, ed. C. S. Kochanek \& J. N. Hewitt (Dordrecht: Kluwer), 189

Garrett, M., Leppänen, K., Porcas, R. W., Patnaik, A. R., Nair, S. \& Teräsranta, H. 1998, in IAU Symp. 164, Radio Emission from Galactic and Extragalactic Compact Sources, ed. J. A. Zensus, G. B. Taylor \& J. M. Wrobel (ASP Conference Series, Vol 144), 313

Jauncey, D., et al. 1991, Nature, 352, 132

King, E. A. 1994, PhD. Thesis, University of Tasmania

Kundić, T., et al. 1997, ApJ, 482, 75

Lovell, J. E. J., et al. 1996, ApJ, 472, L5

Lovell, J. E. J., McCulloch, P. M., King, E. A. \& Jauncey, D. L. 1996a, in IAU 173: Astrophysical Applications of Gravitational Lensing, ed. C. S. Kochanek \& J. N. Hewitt (Dordrecht: Kluwer), 347

Nair, S., Narasimha, D., \& Rao, A. 1993, ApJ, 407, 46

Pelt, J. et al. 1994, A\&A, 286, 775

Pelt, J., Kayser, R., Refsdal, S. \& Schramm, T. 1996, A\&A, 305, 97

Rao, A. \& Subrahmanyan, R. 1988, MNRAS, 231, 229

Shepherd, M. C. 1997, in Astronomical Data Analysis Software and Systems VI, ed. G. Hunt \& H. E. Payne (ASP Conference Series, Vol 125), 77

Tornikoski, M., et al. 1996, A\&AS, 116, 157

Tzioumis, A., et al. 1989, AJ, 98, 36

van Ommen, T., Jones, D., Preston, R., \& Jauncey, D. 1995, ApJ, 444, 561

Wiklind, T. \& Combes, F. 1996, Nature, 379, 139

Wiklind, T. \& Combes, F. 1998, ApJ, 500, 129 




Fig. 1.- (a) The $8.6 \mathrm{GHz}$ light curve data for both components of PKS 1830-211. The measured NE component flux densities are represented by the open triangular symbols and the measured SW component flux densities are represented by the closed circular symbols. (b) The NE and SW component light curves after applying our solutions from the dispersion analysis $(\Delta \tau=-23$ days, $\mu=1.52$ and $d=-0.62 \mathrm{Jy})$. 


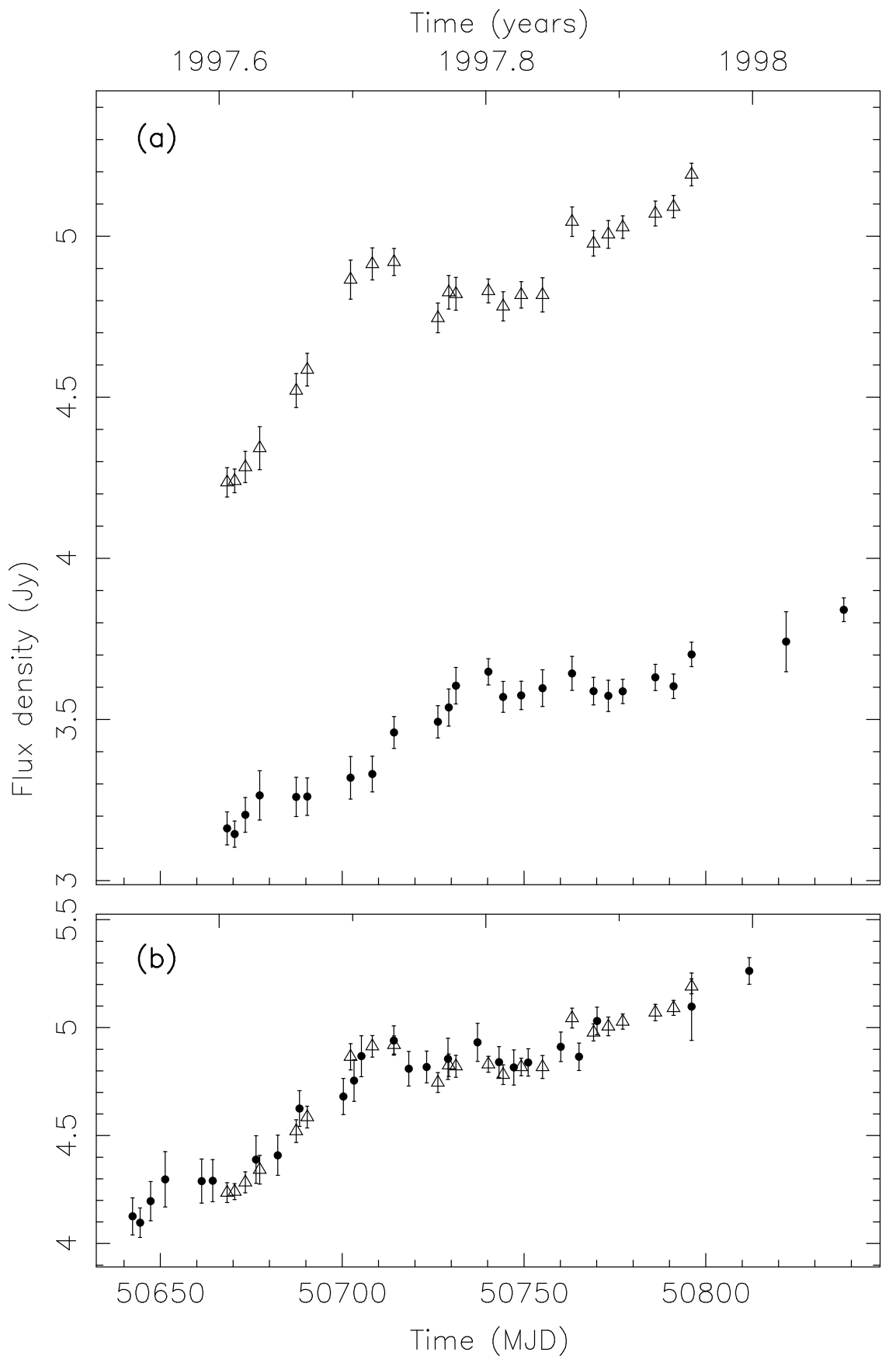

Fig. 2.- (a) The $8.6 \mathrm{GHz}$ light curve data for both components of PKS 1830-211 for the time period surrounding the 'bump' described in Section 2. The measured NE component flux densities are represented by the open triangular symbols and the measured SW component flux densities are represented by the closed circular symbols. (b) The NE and SW component light curves after applying our solutions from the dispersion analysis $(\Delta \tau=-26$ days, $\mu=1.68$ and $d=-1.17 \mathrm{Jy})$. 